\title{
FLUX ENHANCEMENT BY AIR DISPERSION IN CROSS-FLOW MICROFILTRATION OF A COLLOIDAL SYSTEM THROUGH SPIRAL WOUND MODULE
}

\author{
T.M. QAISRANI* \\ W.M. SAMHABER
}

Institute of Process Engineering, A-4060 Leonding, Welser Str. 42, Johannes Kepler University

Linz, Austria

Received: $11 / 02 / 08$

Accepted: 18/03/08 *to whom all correspondence should be addressed: e-mail: tahir.qaisrani@jku.at

\section{ABSTRACT}

Enhancement of the permeate flux due to reduction of cake layer resistance by air-liquid twophase flow in a cross flow spiral wound microfiltration membrane has been investigated. Experiments were carried out with two different suspension systems, namely baker's yeast suspension with a dry mass concentration of $5.4 \mathrm{~kg} \mathrm{~m}^{-3}$ and the colloidal suspension of starch and bacteria having a dry mass concentration of $3.41 \mathrm{~kg} \mathrm{~m}^{-3}$. An air-liquid two-phase flow was generated by injecting the air at the inlet of the membrane module and was fed into a horizontally mounted assembly of a spiral wound microfiltration membrane. The effect of air dispersion was studied on flux enhancement, specific cake layer resistance and loading of cake mass on the membrane surface. This study shows that by air dispersion, the permeate flux can be enhanced up to 60 percent for the colloidal suspension of starch and bacteria while a flux enhancement of 40 percent was achieved for yeast suspension. The specific cake layer resistance reduced by a factor of 10 for both of the suspensions when air was injected into the feed stream. The results of this study depict that the technique of air dispersion is effective in increasing the permeate flux for the suspension systems containing below and above micron sized particulates.

KEYWORDS: Flux enhancement; Air dispersion; Cross flow; Microfiltration; Fine colloids;Spiral wound module

\section{INTRODUCTION}

The application of cross flow MF process is increasing rapidly throughout the world for separation of fine solids from suspensions. This technique has proven to be very effective for suspensions containing very fine solids which can not be separated by traditional separation techniques like settling, sedimentation, centrifugation and dead-end filtration. Concentration, clarification and purification processes in chemical, pharmaceutical, food, beverages \& water processing industries involve processing of suspensions which contain micron and submicron size particles. Cross flow microfiltration and ultrafiltration processes are most suitable for filtration of such suspensions. In these industrial processes, mostly tubular and hollow fiber microfiltration modules have been used for separation of solids from the suspensions. The major draw back of these modules is that the capital cost per unit membrane area is very high as compared to that of spiral wound microfiltration modules available in the market. Also, the spiral wound geometry with open channel spacers is considered to be resistant to fouling due to generation of high level turbulences on membrane surface during the operation. It was, therefore, decided to investigate the effectiveness of air dispersion in fouling control for such microfiltration modules. It is also worthwhile to mention here that this is first time in membrane process research when air dispersion has been used for enhancement of the permeate flux in an MF spiral wound membrane with open channel spacers. Only Cui, 1994 applied air dispersion in an ultrafiltration spiral wound membrane for separation of Dextran. But this work has remained unpublished (Cui et al, 2003). The spiral wound MF elements are widely used 
in the process industry and has been found patented until recently in 2005 in USA (Herron, 2005). Osmonics produced first ever spiral wound element made form Polypropylene in early 80 's with open channel spacers with product code 52T-Y. Figures 1 and 2 below show the end view diagrams of a spiral wound element and open channel spacers.

MF membranes act as physical barrier to the suspended particles ensuring the retention of particles which are greater in size than that of pore size of the membrane. Membrane fouling is considered to be the sole reason for the decline of permeate flux with time in an MF membrane process. Three fouling mechanisms are distinguished in microfiltration process, namely, surface adsorption, cake layer formation \& internal pore blocking. Fouling is a complex phenomenon which is characterized by a decline of permeate flux through the membrane as a result of increase in flow resistance due to one or more fouling mechanisms. The intensity of fouling caused by aforesaid fouling mechanisms is dependent upon various factors like pore size of the membrane, size of the particle, shape of the particle, particle concentration in the suspension, size distribution of particles, membrane material, temperature and the operating conditions of the MF process. The most desired quality in membrane processes is that the membrane should run at a sustainable high flux for a longer period of time. This can be achieved if the membrane fouling is kept to the minimum.

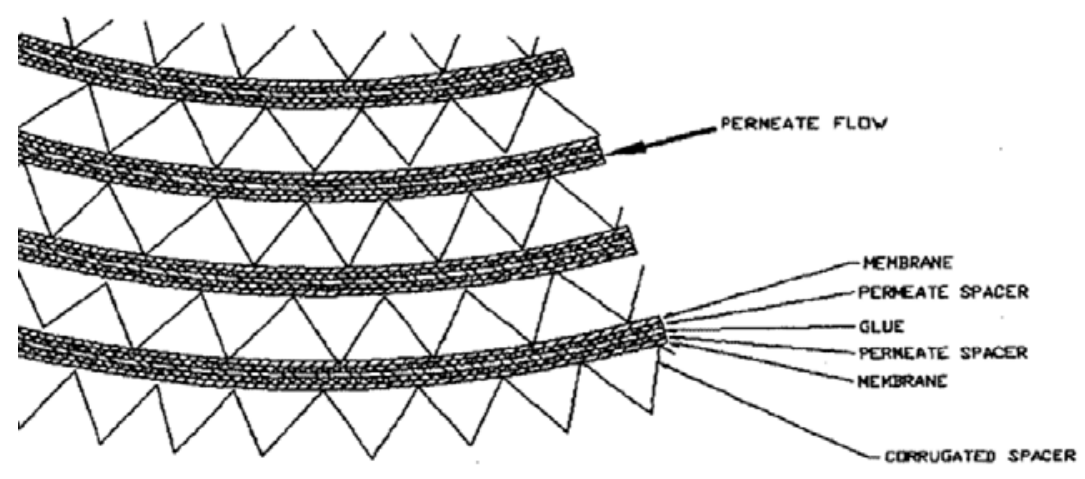

Figure 1. Open channel spacer applied in a spiral wound element

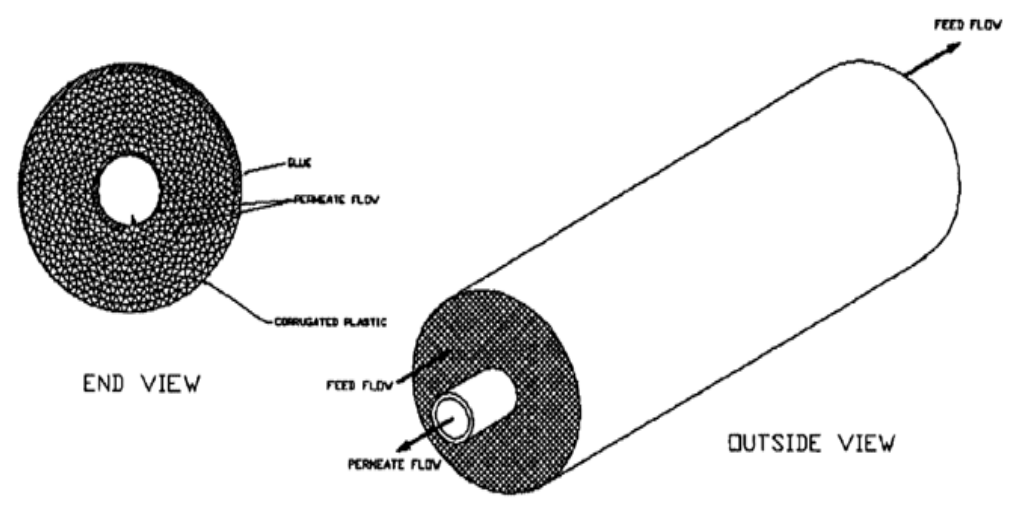

Figure 2. Spiral wound element with open channel spacers

The permeate flux is described as function of resistance as following by Darcy's law:

$$
\mathrm{J}=\frac{\Delta \mathrm{P}}{\mu\left(\mathrm{R}_{\mathrm{m}}+\mathrm{R}_{\mathrm{c}}\right)}
$$


In this equation, $R_{m} \& R_{c}$ are resistances due to membrane and the cake layer deposited on the membrane surface respectively. The membrane resistance $R_{m}$ remains constant, thus making the permeate flux $\mathrm{J}$ dependent upon $\mathrm{R}_{\mathrm{c}}$, the cake layer resistance if all other conditions remain constant. It means that if the membrane fouling can be kept minimum, the permeate flux can be increased.

\subsection{Fouling control by air dispersion}

In an MF process, particle membrane fouling can be controlled if the deposition of particles on the membrane surface is reduced. The deposition of particles on membrane surface is mainly dependent upon the degree of particle-particle and particle-membrane surface adhesion forces. The adhesion forces responsible for deposition of particles on membrane surface are in balance with the shear forces present on membrane surface due to cross flow. It means that if we alter the flow regime from laminar to highly turbulent, the deposition of particles on membrane surface will be decreased to a minimum. Resultantly, the permeate flux can be increased through the membrane. Various researchers have used different turbulence promoting techniques in order to control the fouling on membrane surface. These techniques include: use of turbulence promoters (Finnigan \& Howel,1990); dynamic filtration (Kronev et al); pulsating flows (Gupta et al,1992); jet flows (Arroyo \& Fonade,1993); vortex waves (Milward et al, 1995); baffles as well as electric \& ultrasonic (Kim et al, 2007) and surface \& feed treatments (Racz et al,1986). Although process efficiency can be improved by all aforesaid techniques, but the industrial application of such techniques is limited by technological aspects. Another technique which has attracted the attention of researchers in recent years is continuous injection of gas/air in the feed stream. In this technique, gas/air is dispersed in the liquid stream to generate two-phase flow which produces very high turbulences along the membrane surface. This high turbulence on the membrane surface reduces the deposition of solid particles on membrane surface and resultantly, the permeate flux increases.

Air injection into feed stream to generate a liquid-gas two-phase flow has been found very effective in the permeate flux enhancement in microfiltration processes. Cui and Wright (1994) showed up to a $175 \%$ increase in permeate flux in yeast microfiltration. Mercier et al. (1997) applied slug flow to get a significant increase in the permeate flux with tubular membranes. In another study, Mercier et al. (1998) showed 3 time increase in the permeate flux in ultrafiltration of bentonite and yeast suspension by air sparging/dispersion. All aforementioned researchers used either hollow fiber or tubular modules to investigate the effect of air sparging/dispersion on enhancement of the permeate flux. There is no published work on application of air dispersion for enhancement of permeate flux with spiral wound modules while few researchers have also worked on flat sheet membrane modules with air-liquid twophase flow and were able to enhance the permeate flux with air dispersion(Mercier et al, 2000). Therefore the major objective of this study is to test the technique of air-liquid twophase flow generated by air dispersion in a spiral wound microfiltration element with open channel spacer for the enhancement of the permeate flux.

The spacers in spiral wound assembly create a feed-channel between facing membrane leafs \& promote turbulent flow which reduces fouling phenomena. Starch suspension was selected for these investigations which contained very low concentration of fine particles while yeast suspension was used as a model suspension for the comparison of results.

\subsection{Determination of cake properties}

In classical cake filtration theory, the cake is assumed to be incompressible. Thus successive volumes of filtrate deposit successive layers of cake and each deposited cake-layer having same permeability as that of preceding and following cake layer. The Darcy's law can also be represented as:

$$
\mathrm{J}=\frac{\Delta \mathrm{P}}{\mu\left(\mathrm{R}_{\mathrm{m}}+\mathrm{r}_{\mathrm{c}} \frac{\mathrm{CV}(\mathrm{t})}{\mathrm{A}}\right)}
$$

where $r_{c}$ is flow resistance per unit mass of solid or specific cake layer resistance.

Or 
$\frac{1}{\mathrm{~J}^{2}(\mathrm{t})}-\frac{1}{\mathrm{~J}^{2}(0)}=\frac{2 \mathrm{r}_{\mathrm{c}} \mathrm{c} \mu \mathrm{t}}{\Delta \mathrm{P}}$

where

$$
\mathrm{J}(0)=\frac{\Delta \mathrm{P}}{\mu R_{\mathrm{m}}}
$$

The specific cake layer resistance, i.e., $r_{c}$ can be determined from the slope of the curve of $1 / J^{2}(t)$ as function of time in case of dead-end filtration. But in the case of cross-flow filtration, we ought to know the mass deposited on membrane surface as a function of time. Thus equation 2 can be written as

$$
J(t)=\frac{\Delta P}{\mu\left(R_{m}+r_{c} M(t)\right)}
$$

where $M(t)$ is the amount of solids deposited on membrane surface as a function of time. Therefore, the specific cake resistance can be written as

$r_{c}=\left(\frac{1}{J(t)}-\frac{1}{J(0)}\right) \frac{\Delta P}{\mu M(t)}$

For using equation 6 , the knowledge of amount of solids deposited on membrane surface as a function of time is required. This can be determined in a cross flow experiment by continuously recirculaing permeate and retentate in order to keep the volume of suspension constant and measuring the concentration of solids as a function of time. Equation 7 below then gives us the mass of solids deposited on membrane as function of time

$$
M(t)=V(t) \frac{C_{0}-C_{t}}{A}
$$

\section{EXPERIMENTAL}

\subsection{Materials}

Baker's yeast suspensions and starch containing bacterial strain were used as test suspensions. Yeast is composed of almost spherical particles with a mean diameter of $4.2 \mu \mathrm{m}$ (Sur and Cui, 2001). Yeast was chosen as a model suspension due to its well-defined granulometric properties and for the comparison purpose. The other reason for selecting yeast was its ability to clean the membrane from fine particles of starch which could not be removed from chemical cleaning agents. The yeast suspension was prepared by adding known quantity of yeast in pure water whereas the concentration of starch suspension was determined by dry mass calculation. The starch suspension used was the overflow of a centrifuge waste water recovery unit which indicates that the particles are below micrometer range in the suspension. Yeast was used with a dry mass concentration of $5.4 \mathrm{~kg} \mathrm{~m}^{-3}$ while the concentration of starch was $3.41 \mathrm{~kg} \mathrm{~m}^{-3}$.

\section{$2.2 \quad$ Membrane module}

A bench-mounted spiral wound module was used for the experimentation. PVDF microfiltration elements of type JX 2540 COS from Desalination later named as Osmonics and now, GE Water Technology, USA, with an effective area of $1.01 \mathrm{~m}^{2}$ were used for these tests. The spacer used was of diamond \& ladder shape. The pore size of the membrane was 0.3 $\mu \mathrm{m}$.

\subsection{Experimental apparatus}

Figure 3 illustrates the experimental setup for testing of yeast and starch suspensions. The temperature of the feed tank was kept constant at $22^{\circ} \mathrm{C}$ by continuous tape water circulation in the water jacket of the tank. An eccentric positive displacement pumps was used to circulate the feed. The air was injected at the inlet of the membrane module for ensuring complete mixing of air with the liquid for generating two-phase flow. The inlet and outlet pressures of the membrane modules were measured by two Bourdon pressure gauges. The average of these two measurements gave the trans-membrane pressure.

For turbidity measurements, WTW-Turb 550 turbidity meter was used. The concentration of solids was calculated as function of turbidity. The suspension system was passed through a 5 $\mu \mathrm{m}$ filter before processing through the membrane. Pure water flux was measured and 
recorded before start of each experiment. All experiments were conducted in a recirculation mode.

After each experiment, the system was drained and flushed with de-ionized water. A commercially available enzymatic membrane cleaner, Ultraperm 53 by Hankel was used for cleaning the membrane after microfiltration of yeast suspension while alkaline solution in low concentration was found effective for membrane cleaning after starch filtration.

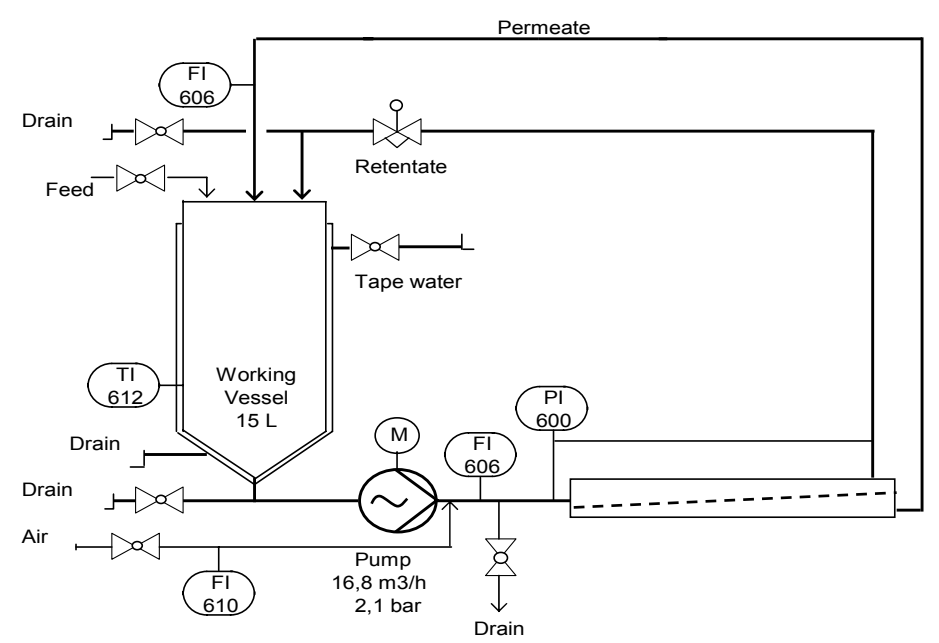

Figure 3. schematic diagram of experimental set up for microfiltration

\section{RESULTS AND DISCUSSION}

\subsection{Effect of air flow rate on permeate flux}

Figures 4(a) and 4(b) below show the effect of air flow rate on the permeate flux for starch and yeast suspensions respectively. From these figures, it can be seen that flux decline is very sharp in case of starch suspension for first 30 minutes of operation as compared to that of yeast suspension.

The flux enhancement is shown in terms of the relative increase in the permeate flux as under:

$\%$ flux enhancement $=\left(\mathrm{J}_{\mathrm{ad}} / \mathrm{J}_{\mathrm{wa}}-1\right) \times 100$

where $J_{a d}$ is the permeate flux with air dispersion where as $J_{w a}$ is the permeate flux with out air dispersion. The permeate flux increased with air dispersion for both of the suspensions. But the enhancement in the permeate flux was found high for the starch-bacteria suspension as compared to that of yeast suspension. For starch-bacteria suspension, an enhancement of about $60 \%$ was observed where as it was around $40 \%$ for the yeast suspension at same operating conditions. The permeate flux kept increasing with increase in air flow rate. This can be due to the difference in size and adhesion properties of the two particle systems. As starch particles are fine being in the range of $100 \mathrm{~nm}$ to $1 \mu \mathrm{m}$, their surface area is very high. Due to high surface area, they exhibit more adhesion to the membrane surface, and are adsorbed on the membrane surface. Therefore in order to take away adsorbed particles, higher magnitude of turbulence was required and air-liquid two-phase flow provided that degree of turbulence. The air dispersion helped to attain the steady state condition quickly and afterwards, the flux remained almost constant throughout the experiment.

\subsection{Effect of air dispersion on mass deposition}

The enhancement in permeate flux by air dispersion can be understood very well if we can see that how air dispersion affects the resistance offered by cake layer deposited on membrane surface with time. The effect of air dispersion on deposition of cake-mass on membrane surface is shown in figure-5. The air dispersion caused more turbulence on membrane surface thus causing a reduction in mass deposited on membrane surface. The quantity of cake-mass is reduced 3 times when air was dispersed in case of yeast suspension whereas it reduced almost two times in case of starch-bacteria suspension. 


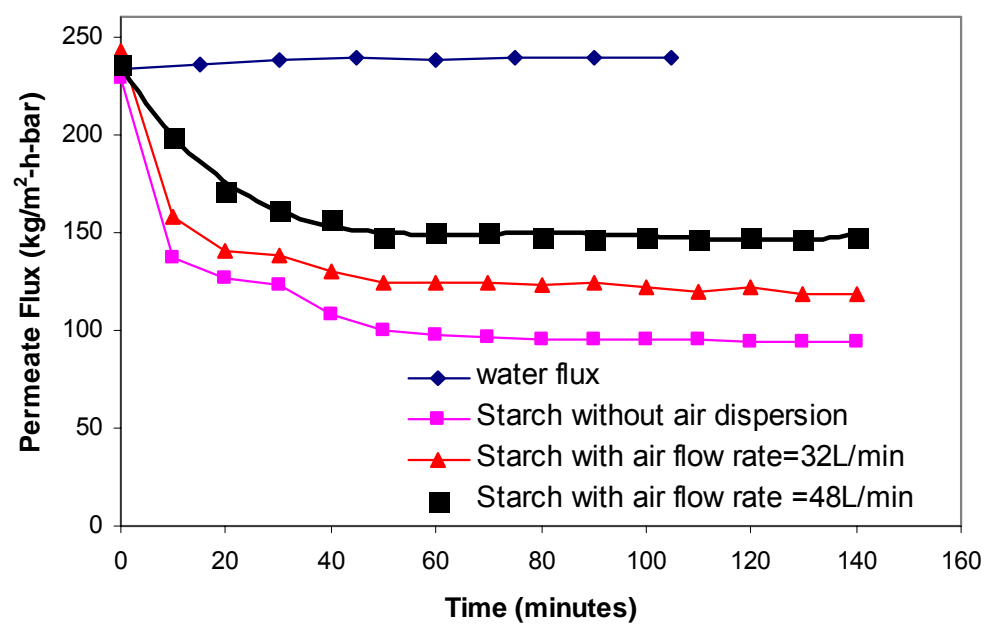

Figure 4(a). Effect of air dispersion on flux decline for starch suspension at $\Delta \mathrm{P}=0.35$ bar; liquid flow rate $=18 \mathrm{I} \mathrm{min}^{-1} ; \mathrm{C}=3.41 \mathrm{~kg} \mathrm{~m}^{-3} ; \mathrm{pH}=2.25$

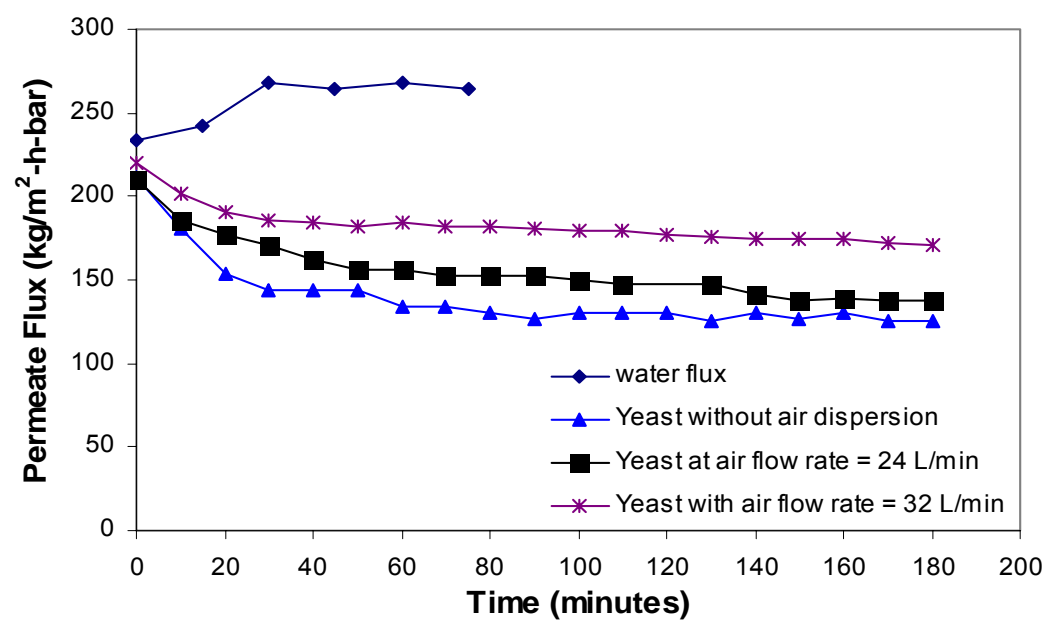

Figure 4(b). Effect of air dispersion on flux decline for starch suspension at $\Delta \mathrm{P}=0.35$ bar; liquid flow rate $=18 \mathrm{I} \mathrm{min}^{-1} ; \mathrm{C}=5,4 \mathrm{~kg} \mathrm{~m}^{-3} ; \mathrm{pH}=2,25$

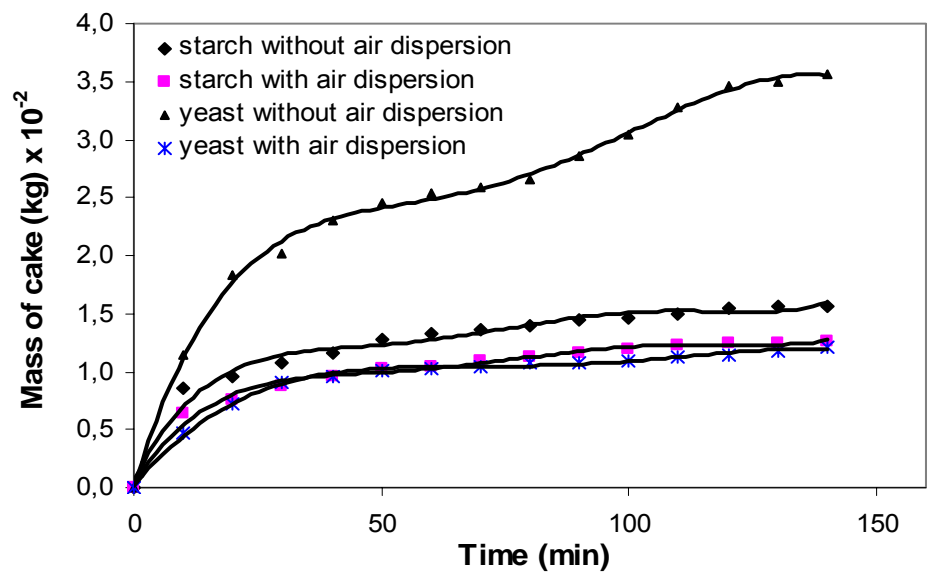

Figure 5. Effect of air dispersion on mass deposition of cake for starch and yeast suspensions at $\Delta P=0.35$ bar and air flow rate $=32 \mathrm{I} \mathrm{min}^{-1}$ 
The cake layer resistance is directly proportional to the cake-mass deposited on the membrane surface as shown in equation 5 . Therefore, with reduction in cake-mass deposition on membrane surface, the cake layer resistance decreased and resultantly, the enhancement in flux was achieved with air-liquid two-phase flow for both the suspension systems. Also air dispersion caused a steady rate of mass deposition on membrane surface which helped the steady-state condition to reach earlier.

\subsection{Effect of air dispersion on cake resistance}

Figures 6 and 7 below show the effect of air dispersion on specific resistance of the cake with time. For both systems of suspensions, that is, starch-bacteria and yeast suspensions, the resistance decreased to an order of 10 when air was dispersed in the suspension.

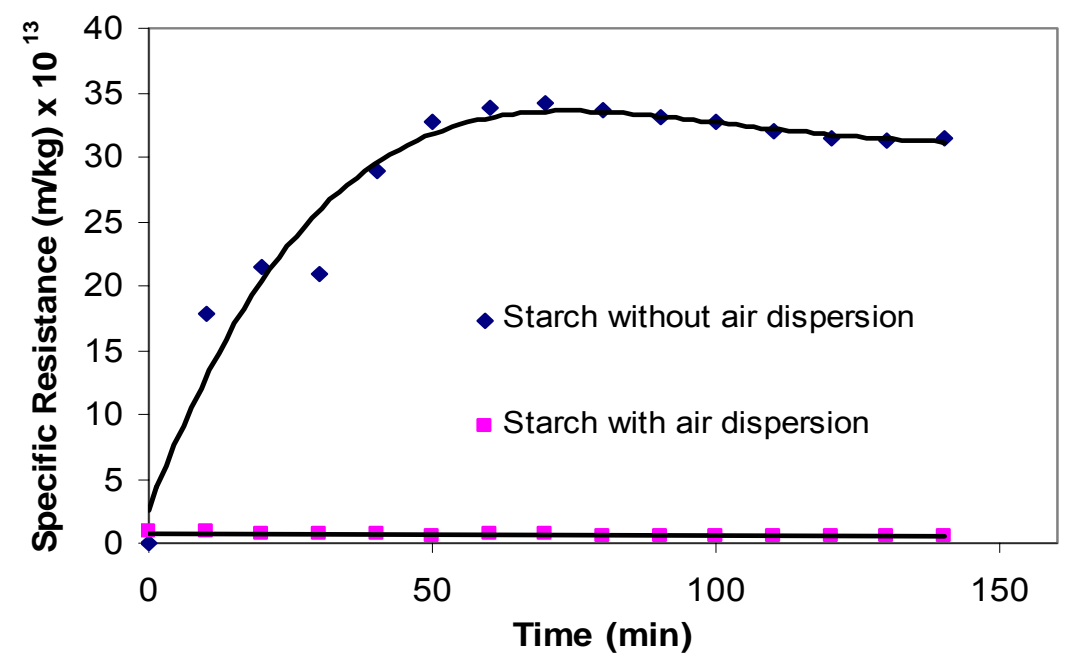

Figure 6. Effect of air dispersion on specific cake resistance for starch at $\Delta \mathrm{P}=0.35$ bar and air flow rate $=32 \mathrm{I} \mathrm{min}^{-1}$

Both starch and yeast suspensions exhibited similar behavior for decrease in cake resistance in presence of air dispersion. The cake layer resistance for yeast suspension was found to be significantly low as compared to that of starch suspension. This can be due to difference in particle size. The yeast suspension consisted of particles with more than $4 \mu \mathrm{m}$ size while starch particles were in nanometer size range. This means that cake formed by yeast cells might be porous due to low surface area of the particles where as the cake formed by starchbacteria particles should be very dense. Thus a low cake layer resistance was observed for yeast suspension.

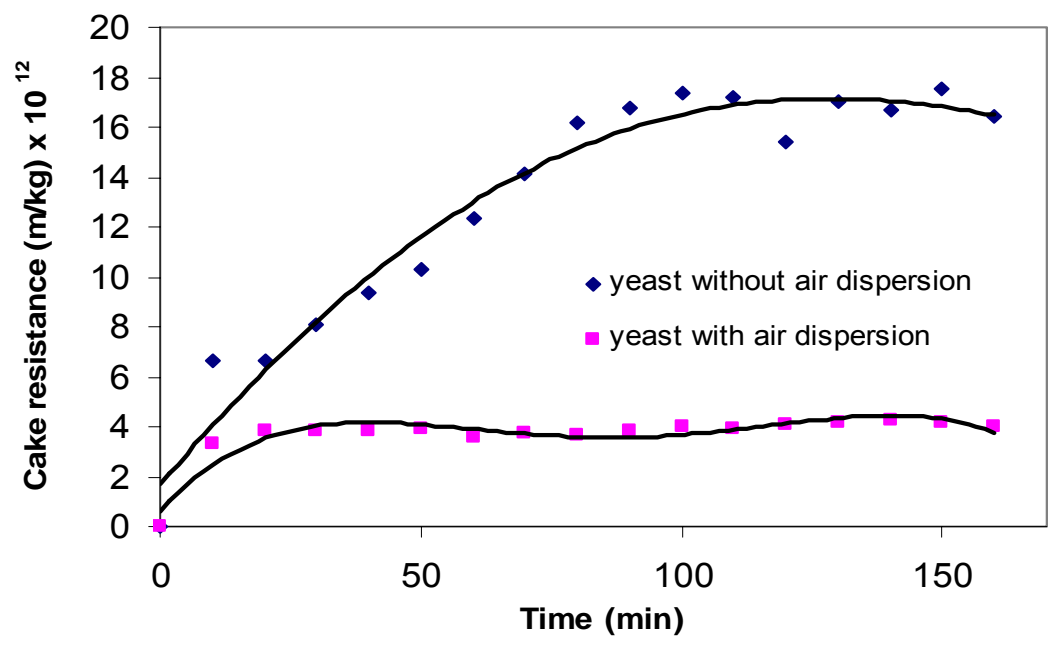

Figure 7. Effect of air dispersion on specific cake resistance for yeast at $\Delta \mathrm{P}=0.35$ bar and air flow rate $=32 \mathrm{I} \mathrm{min}^{-1}$ 


\section{CONCLUSIONS}

From this experimental study, the following conclusions can be deduced:

1. Air dispersion is effective in enhancing the permeate flux in an open channel spiral wound microfiltration membrane module for suspensions having particles with size below and above micron range.

2. Air dispersion is more effective for severe fouling system, that is, for suspension having very fine colloids.

3. The permeate flux increases at all air flow rates for both the suspension systems

4. The mechanism of fouling reduction in by air injection in a spiral wound element with open channel spacers needs to be investigated.

\section{LIST OF SYMBOLS}

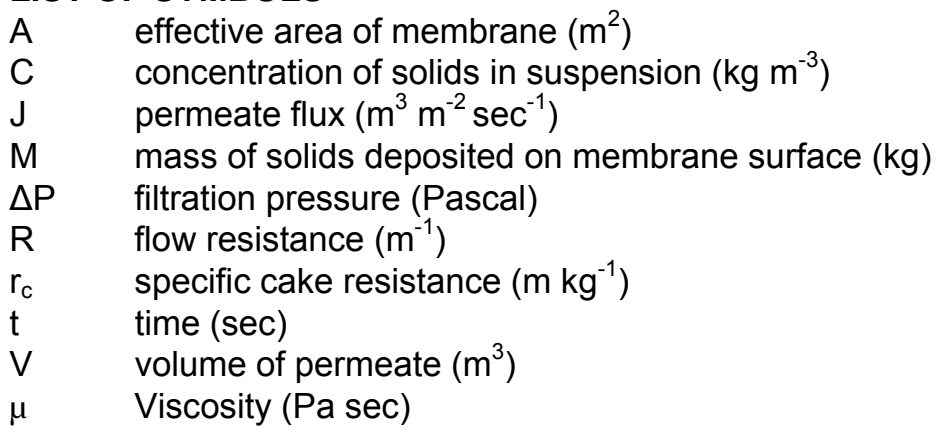

\section{REFERENCES}

Arroyo G., Fonade C., (1993), Use of intermittent jets to enhance flux in cross flow filtration, J. Memb. Sci., 80, 117-129.

Cabassud C., Laborie S. and Laine JM., (1997), How slug flow can improve ultrafiltration in organic hollow fibers, J. Memb. Sci., 128, 93-101.

Cui Z.F., Chang S. and Fane A.G., (2003) The use of gas bubbling to enhance membrane processes, J. Memb. Sci., 221, 1-35.

Cui Z.F. and Wright K.I.T., (1994), Air sparging to enhance microfiltration in waste water treatment, Engineering in Membrane processes II-Environmental Applications II, Ciocco, Italy, April 2628.

Finnigan S.M., Howel J.A., (1990) The effect of pulsatile flow on UF fluxes in a baffled tubular membrane system, Desalination, 79, 181-202.

Gubta B.B., Blanpin P., Jaffrin M.Y., (1992), Permeate flux enhancement by pressure \& flow pulsations in microfiltration with mineral membrane, J. Memb. Sci., 70, 257-266.

Herron J., (2005), Open-channeled spiral wound membrane module, Patent No: US 6,902, 672 B2, June 7, 2005, United States Patent, 1-9 (http://www.freepatentsonline.com/6902672.pdf)

Kim J.O., Jung J.T., Yeom I.T., \& Aoh G.H., (2007), Electric fields treatment for reduction of membrane fouling, the inactivation of bacteria \& the enhancement of particle coagulation, Desalination, 202, 31-37.

Kronev K.H., Nissinen V., Ziegler H., (1987), Improved dynamic filtration of microbial suspension, Biotechnology, 5, 921-926.

Ma H., Bowman C.N., Davis R.H., (2000), Membrane fouling reduction by back pulsing \& surface modification, J. Memb. Sci., 173, 191-200.

Mercier M., Fonade C., Lafforgue-Delorme C., (1997), How slug flow can enhance the ultrafiltration flux in mineral tubular membranes, J. Memb. Sci., 128, 103-113.

Mercier M., Lagane C., Fonade C., (2000), Influence of gas/liquid two-phase flow on the ultrafiltration and microfiltration performances: case of a ceramic flat sheet membrane, $\mathrm{J}$. Memb. Sci., 180, 93-102.

Mercier M., Maranges C. and Fonade C., (1998), Yeast suspension filtration, flux enhancement using an upward gas/liquid slug flow-application to continuous alcoholic fermentation with cell recycle, Biotech \& Bioengg, 58, 47-57.

Millward H.R., Bellhonse B.J., Sobey I.J., and Lewis R.W.H.,(1995), Enhancement of plasma filtration using the concept of the vortex wave, J. Memb. Sci., 100, 121-129. 
Racz I.G., Wassink J.G., Klassen R., (1986), Mass transfer, fluid flow \& membrane properties in flat \& corrugated plate hyper filtration modules, Desalination, 60, 213-222

Sur H.W., \& Cui Z.F., (2001), Experimental study on the enhancement of yeast microfiltration with gas sparging, J. Memb. Sci., 76, 477-484.

Vera L., Villarreal R., Delgado S. and Elmaleh S., (2000) Enhancing microfiltration through an inorganic tubular membrane by gas sparging, J. Memb. Sci., 165, 47-57. 